\title{
A Review of Low-Intensity Transcranial Focused Ultrasound for Clinical Applications
}

\author{
A. Bystritsky • A. S. Korb
}

Published online: 7 March 2015

(C) Springer International Publishing AG 2015

\begin{abstract}
The field of therapeutic focused ultrasound neuromodulation has made great advances in the last few years. While no clinical trials of focused ultrasound neuromodulation are yet underway, several human experiments have recently been conducted. There are many potential uses of this new technology, including treatment of numerous psychiatric and neurologic disorders, as well as a brainmapping tool for discoveries in basic science. In this review, we examine recent research data on the use of focused ultrasound in neuronal tissue, animal models, and humans. We also investigate ideal parameters for neuromodulation as well as potential mechanisms.
\end{abstract}

Keywords Focused ultrasound · Neuromodulation · BRAIN $\cdot$ Imaging $\cdot$ Treatment

\section{Introduction}

Therapeutic focused ultrasound uses low energy sound waves that pass through the skin and skull without surgery, and can be focused with precision essentially anywhere in the brain to modulate neural activity. This type of highly targeted, yet non-invasive, neuromodulation offers the possibility of new

This article is part of the Topical Collection on Neuromodulation

A. Bystritsky • A. S. Korb

Semel Institute for Neuroscience and Human Behavior,

David Geffen School of Medicine, University of California,

Los Angeles, CA, USA

\section{A. Bystritsky $(\bowtie)$}

Semel Institute for Neuroscience and Human Behavior,

David Geffen School of Medicine, 300 UCLA Medical Plaza, 2335,

Los Angeles, CA 90095, USA

e-mail: abystritsky@mednet.ucla.edu therapies for numerous neurologic and psychiatric conditions including epilepsy, depression, anxiety disorders, and traumatic brain injury. While no clinical trials of therapeutic focused ultrasound neuromodulation have yet been conducted, in the past few years, it has moved even closer to becoming a reality.

A few years ago, we wrote a review summarizing the state of focused ultrasound neuromodulation, arguing that the field was ready for first-in-human studies. Experiments in multiple animal models demonstrate that focused ultrasound (FUS) is highly focused, safe, and effective at neuromodulation. Subsequently, several studies have been published on focused ultrasound neuromodulation in humans.

The need for a technology like FUS is large and other noninvasive neuromodulation techniques - such as repetitive transcranial magnetic stimulation (rTMS) and transcranial direct current stimulation (TDCS) - are beginning to be utilized more broadly for treatment of neurologic or psychiatric disorders. Other forms of non-invasive neuromodulation-such as electroconvulsive therapy (ECT) - have been used for decades. However, these all suffer from limitations in terms of either spatial specificity, or are not useful as a general tool for neuromodulation. A general tool for neuromodulation may not only lead to new therapies but also new ways of diagnosing as well as opening new pathways for scientific discovery.

rTMS cannot be focused in three dimensions, and thus is limited to superficial targets. Similarly, TDCS also cannot be focused, nor can ECT. And while rTMS and TDCS appear to have many general applications, ECT, while very effective at treating depression, does not appear to generalize to other applications.

In contrast to other technologies, ultrasound (US) can be focused in three dimensions in a highly targeted manner. It also appears to not be disease specific and thus generalizable to many different conditions. FUS's ability to precisely modulate region-specific brain activity may translate into safe and long-lasting therapeutic applications. 
Repeated use of suppressive FUS may have a long-term effect, just as repeated use of TMS can have a long-term neuromodulating effect in depression. We envision that after using an MRI for initial targeting, subsequent treatment can be done in a doctor's office.

There are many potential uses of this exciting new technology. Aside from treating disorders, it is possible that FUS could be used in pre-surgical mapping as well as diagnosis of various disorders, and as a brainmapping tool for discoveries in basic science. The last several years have seen great advances in expanding applications, understanding of mechanisms, and even the first human testing (Table 1).

\section{FUS Neuromodulation in Humans}

In our previous review [14], we discussed the early evolution of focused ultrasound neuromodulation, beginning with the first attempts to study ultrasound's effect on neuronal tissue in the 1920s [15] and progressing through until today. Even nearly 60 years ago, Fry predicted that focused ultrasound (US) would have a major impact on neurology, including surgical treatments [16], as well as for investigating structure and function of brain circuitry [17]. While early studies of focused ultrasound primarily centered on high-intensity ultrasound for tissue ablations, in the last decade, there has been a surge in research on low-intensity focused ultrasound, not for surgery but for neuromodulation.

The neuromodulatory effects of FUS have been demonstrated numerous times in recent studies in multiple animal models. Based on pulse parameters, studies have shown that FUS can stimulate or suppress neural activity. FUS stimulation previously discussed includes stimulation of hippocampal slices [18], as well as motor cortex [19]. FUS has also been shown to suppress visual-evoked potentials [19], and even epileptic activity [20]. These varied effects and applications illustrate the potential of low-intensity focused ultrasound pulsation (LIFUP) to be a general neuromodulation tool.

Furthermore, and perhaps most importantly, FUS can be effective at neuromodulation without causing tissue damage $[12,18,19,21]$. No studies have shown that FUS induced tissue damage in the absence of heating, unless they utilized contrast agents to enhance cavitation effects [14]. Therefore, FUS appears safe, even at intensities several times higher than the FDA limit for diagnostic ultrasound $(720 \mathrm{~mW} / \mathrm{cm},[15])$.

Based on the safety profile of FUS, in our previous review, we recommended that human experiments should be conducted. Subsequently, three ultrasound neuromodulation experiments in humans have been reported within the last 2 years.

One human study at the University of Arizona looked at the therapeutic use of transcranial ultrasound on mood and affect. This study utilized a standard clinical ultrasound device. While they did not specifically use focused ultrasound, the results may still be applicable [ $1 \bullet]$. Participants were volunteer patients suffering from chronic pain. The ultrasound probe was applied by a physician to the scalp over the posterior frontal cortex, contralateral to maximal pain. The ultrasound machine itself was operated by a separate investigator, which allowed this study to be conducted in a double-blind fashion. Transcranial ultrasound was administered in standard B-mode for $15 \mathrm{~s}$. Before and after treatment, subjects completed subjective reports on pain and mood. All subjects received both US and placebo in a randomized order. The results showed that brief US exposure led to improvement in mood and global affect that persisted for at least $40 \mathrm{~min}$.

A second set of studies on humans examined the effect of transcranial focused ultrasound on evoked potentials, and the ability to enhance sensory discrimination. In these studies, FUS was administered to the scalp over somatosensory cortex during concurrent stimulation of the median nerve. The results showed that FUS significantly decreased amplitude of several stimulus-evoked potentials [2•]. In addition, FUS altered EEG dynamics of intrinsic EEG activity as well as in evoked potentials in a frequency-band dependent manner [22]. These results illustrate that FUS stimulation can modulate brain electrical activity.

This study also demonstrated that FUS neuromodulation of somatosensory cortex had an effect on perception. When subjects were asked to discriminate between touch stimuli on their hands, FUS improved both spatial and temporal discrimination.

Importantly, this study did not report any adverse events despite using a spatial-peak, temporal-average intensity $\left(\mathrm{I}_{\text {spta }}\right)$ of $8.6 \mathrm{~W} / \mathrm{cm}$ [15], which is an order of magnitude greater than the FDA limit for $\mathrm{I}_{\text {spta }}$ for diagnostic US imaging of $720 \mathrm{~mW} /$ $\mathrm{cm}$ [15]. Although, the spatial-peak pulse-average intensity ( $\mathrm{I}_{\text {sppa }}$ ) of $23.87 \mathrm{~W} / \mathrm{cm}[15]$ is well below the FDA limit for diagnostic US imaging of $190 \mathrm{~W} / \mathrm{cm}$ [15]. The study cautiously utilized short duration sonications $(0.5 \mathrm{~s})$ in order to prevent thermal damage. However, these FDA limits are for diagnostic US imaging only. No such limits exist for FUS neuromodulation. Because the FDA does not have predefined limits for FUS neuromodulation, these data are useful in helping determine what FUS doses can be considered safe.

Recently, Yoo [23] presented a third human study that targeted somatosensory cortex. All subjects participating in the study reported sensations of movement. The results further demonstrate the ability of FUS neuromodulation to affect human perceptions.

While all of the above studies aimed to target specific locations in the human brain, none of them utilized functional imaging as a confirmation that the target region was affected. The lack of functional imaging makes it difficult to document where the focus of stimulation was located, and further show that activity in this region was in fact modulated. While the study from Legon et al., which utilized EEG, provided some 


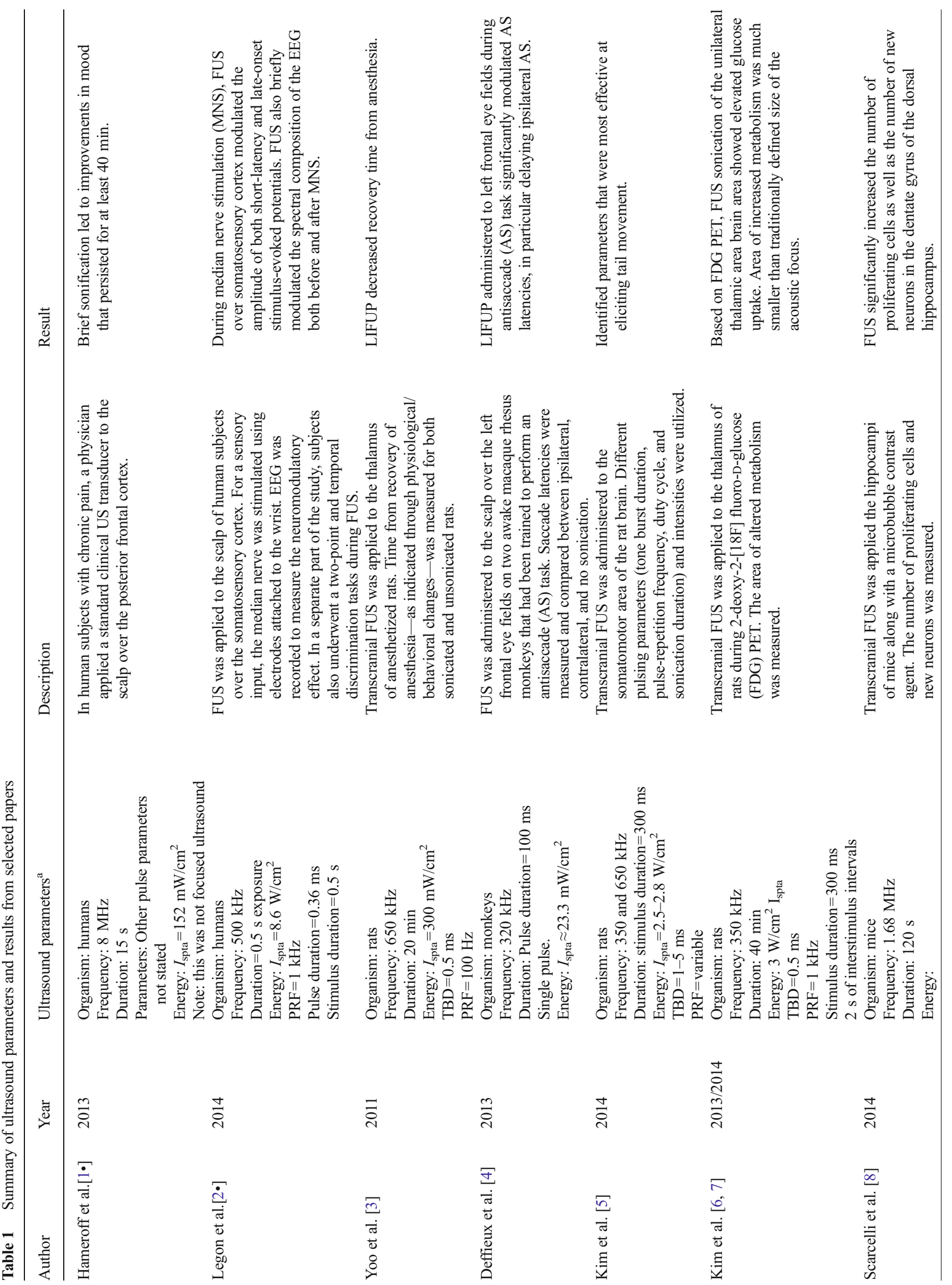




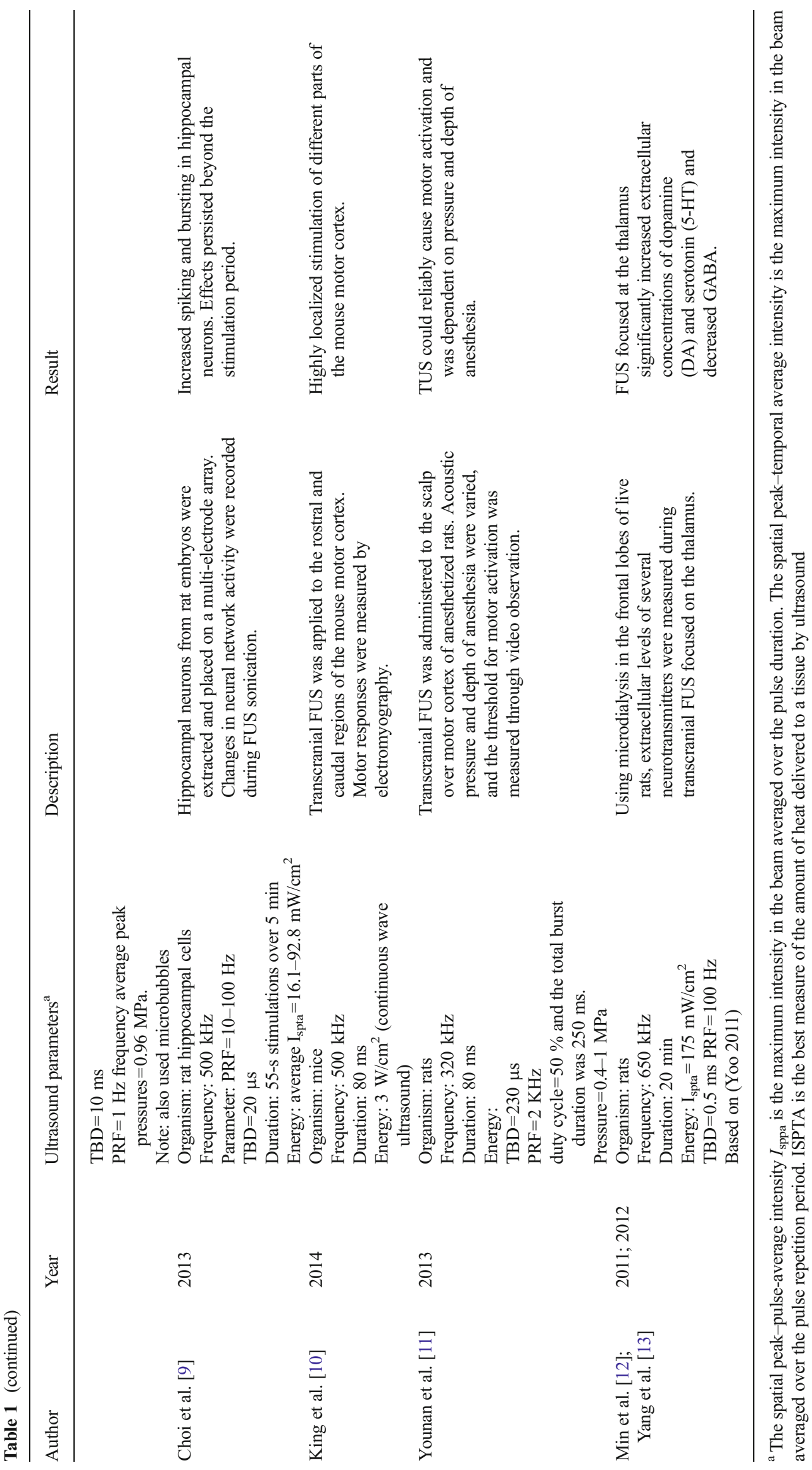


amount of this information, EEG does not have a good 3D spatial resolution. Further studies would benefit from utilizing MR guidance with fMRI feedback to clarify targeting and document the effect of neuromodulation.

\section{Refining Parameters and Expanding Applications}

While human experiments have shown the feasibility of transcranial focused ultrasound neuromodulation in humans, animal experiments continue to clarify ranges of usefulness of FUS parameters in different animal models using a variety of methodologies.

Research has even extended to non-human primates. In macaques, FUS administered to the left frontal eye fields during an antisaccade (AS) task significantly modulated AS latencies, in particular delaying ipsilateral AS [4].

Animal work has also demonstrated even wider-ranging applications for focused ultrasound. In anesthetized rats, FUS applied to thalamus decreased the time to emergence of voluntary movement as well as reflexive response to pinch [3]. This suggests that FUS may be useful in treating disorders of consciousness such as vegetative state.

FUS can also help grow new neurons. In one study, focused ultrasound with microbubbles increased hippocampal neurogenesis in adult mice [8]. This has implications for any neurodegenerative disorder, and particularly Alzheimer's. Other studies have also shown that FUS can even impact neural cell growth and morphology [24].

More realistic models have led to better approximation of focal pressure and size [25], and animal work has demonstrated that FUS can have excellent targeting. For example, focused ultrasound in rats caused increase in glucose metabolism with high spatial specificity [6]. In addition, while the size of the acoustic focus is generally described as the fullwidth at half maximum (FWHM), this same group found that the neuromodulatory area of FUS is much more localized, and is better approximated to be full-width at $90 \%$ maximum. The neuromodulatory area was $3.7 \mathrm{~mm}$ in cross-sectional diameter and $5.6 \mathrm{~mm}$ long, compared to the FWHM, which was $6.5 \mathrm{~mm}$ in diameter and $24 \mathrm{~mm}$ in length. Thus, the neuromodulatory area was almost half the diameter and onefourth the length of the conventional size of the acoustic focus [7]. Even within the tiny mouse motor cortex, it is possible to stimulate rostral and caudal regions separately [10].

Due to physical principals, the lower the frequency of US, the larger the focal area. And yet, higher frequency US signal experience severe attenuation by the skull. One group found a clever possible workaround. Using two transducers of approximately $2 \mathrm{MHz}(2.25$ and $1.75 \mathrm{MHz}$ ), they were able to create "modulated focused ultrasound," which had an effective frequency of $500 \mathrm{kHz}$, but a very small focus [26]. They were able to modulate the mouse brain with very high spatial specificity. However, while this is an interesting technique, it may not be as effective in human applications, as frequencies above approximately $700 \mathrm{kHz}$ get extremely attenuated by the human skull.

While high spatial specificity is clearly evident, there is still a wide disagreement about the minimum intensity necessary for neuromodulation. One group stimulated the somatomotor areas of the rat brain to observe tail movement. Despite systematically altering several parameters, including tone burst duration, center frequency of the ultrasound transducer, duty cycle, and stimulus duration, the lowest effective $I_{\text {spta }}$ was $2.5 \mathrm{~W} / \mathrm{cm}$ [15], which is still 3.5 times higher than the FDA limit [5]. It is becoming more and more clear that FUS neuromodulation has a mechanical mechanism, and is thus pressure dependent [11]; yet it is unclear what the ideal pressures and intensities are. While several groups find that neuromodulation requires stimulation above the FDA diagnostic intensity limit [10], several other groups have achieved effective stimulation below the limit of $720 \mathrm{~mW} / \mathrm{cm}[1 \bullet, 3,12$, $13,15]$, and some have found that much lower intensities still work, even well below $720 \mathrm{~mW} / \mathrm{cm}[15,4,9]$. While depth of anesthesia likely plays a role [11], it cannot fully explain the wide disparity in values. Nor can it be explained by transcranial attenuation.

There is also a disagreement about the relative effectiveness of pulsed vs continuous stimulation. While most groups used pulsed sonication, one group found that continuous sonication was slightly more effective [27]. Although for continuous US their sonication durations were quite short, ranging from 20 to $480 \mathrm{~ms}$. However, regardless of the ideal parameters for FUS, all these studies agree that effective neuromodulation can be achieved without tissue damage.

\section{Mechanism of Neuromodulation}

Several studies have been conducted to clarify mechanisms of action of focused ultrasound neuromodulation. The neuromodulatory effect appears to be mediated through mechanical interaction with the tissue [28]. In one study, focused ultrasound was used to modulate conduction of action potentials along an axon. This study showed that action potential amplitude and velocity were reduced proportional to the cumulative radiation force, thus pointing to a mechanical mechanism.

In particular, the neuromodulatory effect likely comes through cavitation within the lipid bilayer of the neuron cell membrane $[29,30]$. Studies suggest that the physical pressure changes of the ultrasound beam actually move the lipid bilayer, and altering the space within bilayers, causing changes in membrane capacitance. Additionally, other fluid-mechanical properties may also play a role [31]. 
Some evidence suggests that FUS causes direct activation of neurons and synaptic vesicle release [21], while other evidence suggests that it does not directly activate neurons but rather increases neuronal excitability [9]. Further work is necessary to determine the exact effect of TUS on neuronal activity.

Neurochemical changes are also important to consider. While changes in neurochemistry may not be the primary mode of action of FUS, its effects on membranes alter the release of neurotransmitters. Evidence shows that FUS can modulate levels of various neurotransmitters. Using microdialysis, combined with FUS focused on the thalamus of rats, two studies from the same group demonstrated that FUS increased the concentrations of extracellular dopamine (DA) and serotonin (5-HT) [12] while decreasing extracellular GABA [13].

\section{Conclusion}

We need to continue with animal experiments that can clarify parameters, mechanisms of actions, and possible but yet unknown hazards of FUS use. However, we need to proceed with carefully designed safety and efficacy studies that could be conducted in populations where possible future benefits outweigh the risks. Some of those studies that stay under the FDA limits for diagnostic US could be conducted under IRBs supervision as in Hameroff, Yoo, and Legon studies. A human clinical trial is currently under way at UCLA testing the safety of a single-element transducer. Although, new generations of brain-stimulating FUS devices, possibly utilizing multi-array designs, may offer better targeting.

Initial targeting may need to use structural and functional MRI to document the focus position and response within the brain. It is possible to do targeted focused ultrasound outside an MRI environment using MRI data and optical tracking [32]. While these methods were developed with rodents, they could easily be translated to humans. This type of image guidance offers the possibility of multiple FUS treatments in an office setting, not requiring an MRI, improving the feasibility of repetitive FUS similar to rTMS.

Despite the exciting possibilities of clinical trials, so far, no focused ultrasonic neuromodulation devices have yet been approved by the FDA. The approval process will most likely be tedious depending on the ultrasound intensity necessary for effective neuromodulation or brainmapping. So far, human experiments have utilized intensities under the FDA guideline for diagnostic ultrasound and were subthermal. If the intensities can stay under the FDA limits for diagnostic ultrasound, the process will likely be shorter.

It would be helpful to clearly differentiate the different types of therapeutic focused ultrasound. Low-intensity focused ultrasound pulsation (LIFUP) is administered intermittently and subthermally for the purpose of neuromodulation. By contrast, high-intensity focused ultrasound (HIFU) is administered continuously and produces heating of the brain tissue utilized in surgical ablation. The current studies suggest that LIFUP could be used in humans therapeutically. However, if intensities need to be above the FDA guidelines for diagnostic US or will become thermally noxious (e.g., increase regional brain temperature by $2-3^{\circ} \mathrm{C}$ ), the safety of human experiments will need to be thoroughly evaluated and possibly the FDA and the scientific community would need to develop new safety guidelines for therapeutic neuromodulatory focused ultrasound.

\section{Compliance with Ethics Guidelines}

Conflict of Interest Alexander Bystritsky reports other from Brainsonix, during the conduct of the study, outside the submitted work. In addition, Dr. Bystritsky has several patents with royalties paid and a major stock holder of Brainsonix corporation. He also has several patents or pending patents. Alexander Korb reports grants from The Gerald J and Dorothy R Friedman NY Foundation for Medical Research, and personal fees from the Pacific Institute of Medical Research, during the conduct of the study.

Human and Animal Rights and Informed Consent This article does not contain any studies with human or animal subjects performed by the author.

\section{References}

Papers of particular interest, published recently, have been highlighted as:

- Of importance

1. Hameroff S, Trakas M, Duffield C, Annabi E, Gerace MB, Boyle P, et al. Transcranial ultrasound (TUS) effects on mental states: a pilot study. Brain Stimulation. 2013;6:409-15. This study shows that ultrasound in humans, applied at the scalp over posterior frontal cortex, has the potential to enhance mood.

2. Legon W, Sato TF, Opitz A, Mueller J, Barbour A, Williams A, et al. Transcranial focused ultrasound modulates the activity of primary somatosensory cortex in humans. Nat Neurosci. 2014;17: 322-9. This study demonstrates that FUS in humans, applied at the scalp over somatosensory cortex, can modulate the EEG signal. In a separate task, touch discrimination was also affected.

3. Yoo SS, Kim H, Min BK, Franck E, Park S. Transcranial focused ultrasound to the thalamus alters anesthesia time in rats. Neuroreport. 2011;22:783-7.

4. Deffieux T, Younan Y, Wattiez N, Tanter M, Pouget P, Aubry JF. Low-intensity focused ultrasound modulates monkey visuomotor behavior. Curr Biol: CB. 2013;23:2430-3.

5. Kim H, Chiu A, Lee SD, Fischer K, Yoo SS. Focused ultrasoundmediated Non-invasive brain stimulation: examination of sonication parameters. Brain Stimulation. 2014;7:748-56.

6. Kim H, Park MA, Wang S, Chiu A, Fischer K, Yoo SS. PETCT imaging evidence of FUS-mediated (18)F-FDG uptake changes in rat brain. Med Phys. 2013;40:033501. 
7. Kim H, Lee SD, Chiu A, Yoo SS, Park S. Estimation of the spatial profile of neuromodulation and the temporal latency in motor responses induced by focused ultrasound brain stimulation. Neuroreport. 2014;25:475-9.

8. Scarcelli T, Jordao JF, O'Reilly MA, Ellens N, Hynynen K, Aubert I. Stimulation of hippocampal neurogenesis by transcranial focused ultrasound and microbubbles in adult mice. Brain Stimulation. 2014;7:304-7.

9. Choi JB, Lim SH, Cho KW, Kim DH, Jang DP, Kim IY. The effect of focused ultrasonic stimulation on the activity of hippocampal neurons in multi-channel electrode. I Ieee Embs C Neur E. 2013: 731-734.

10. King RL, Brown JR, Pauly KB. Localization of ultrasound-induced in vivo neurostimulation in the mouse model. Ultrasound Med Biol. 2014;40:1512-22.

11. Younan Y, Deffieux T, Larrat B, Fink M, Tanter M, Aubry JF. Influence of the pressure field distribution in transcranial ultrasonic neurostimulation. Med Phys. 2013;40:082902.

12. Min BK, Yang PS, Bohlke M, Park S, Vago DR, Maher TJ, et al. Focused ultrasound modulates the level of cortical neurotransmitters: potential as a new functional brain mapping technique. Int $\mathrm{J}$ Imaging Syst Technol. 2011;21:232-40.

13. Yang PS, Kim H, Lee W, Bohlke M, Park S, Maher TJ, et al. Transcranial focused ultrasound to the thalamus is associated with reduced extracellular GABA levels in rats. Neuropsychobiology. 2012;65:153-60.

14. Bystritsky A, Korb AS, Douglas PK, Cohen MS, Melega WP, Mulgaonkar AP, et al. A review of low-intensity focused ultrasound pulsation. Brain Stimulation. 2011;4:125-36.

15. Harvey EN. The effect of high frequency sound waves on heart muscle and other irritable tissues. Am J Physiol. 1929;91:284-90.

16. Fry WJ, Barnard JW, Fry FJ, Brennan JF. Ultrasonically produced localized selective lesions in the central nervous system. Am J Phys Med. 1955;34:413-23.

17. Fry WJ, Dunn F. Some aspects of the present status of ultrasound in fundamental biological research and in medicine (A Report on the International Conference of Ultrasonics in Medicine, Los-Angeles, California, September 6-7, 1957). J Acoust Soc Am. 1957;29: 1374-5.

18. Tufail Y, Matyushov A, Baldwin N, Tauchmann ML, Georges J, Yoshihiro A, et al. Transcranial pulsed ultrasound stimulates intact brain circuits. Neuron. 2010;66:681-94.

19. Yoo SS, Bystritsky A, Lee JH, Zhang Y, Fischer K, Min BK, et al. Focused ultrasound modulates region-specific brain activity. Neuroimage. 2011;56:1267-75.
20. Min BK, Bystritsky A, Jung KI, Fischer K, Zhang Y, Maeng LS, et al. Focused ultrasound-mediated suppression of chemically-induced acute epileptic EEG activity. BMC Neurosci. 2011;12:23.

21. Tyler WJ, Tufail Y, Finsterwald M, Tauchmann ML, Olson EJ Majestic $\mathrm{C}$, et al. Remote excitation of neuronal circuits using low-intensity, low-frequency ultrasound. PloS one. 2008;3.

22. Mueller J, Legon W, Opitz A, Sato TF, Tyler WJ. Transcranial focused ultrasound modulates intrinsic and evoked EEG Dynamics. Brain Stimulation. 2014.

23. Yoo SS. Paper presented at the 4th International Focused Ultrasound Symposium. Washington; 2014.

24. Hu Y, Zhong W, Wan JM, Yu AC. Ultrasound can modulate neuronal development: impact on neurite growth and cell body morphology. Ultrasound Med Biol. 2013;39:915-25.

25. Metwally MK, Han HS, Jeon HJ, Khang G, Kim TS. Influence of the anisotropic mechanical properties of the skull in low-intensity focused ultrasound towards neuromodulation of the brain. Conference proceedings:... Annual International Conference of the IEEE Engineering in Medicine and Biology Society. IEEE Engineering in Medicine and Biology Society. Annual Conference 2013. p 4565-4568.

26. Mehic E, Xu JM, Caler CJ, Coulson NK, Moritz CT, Mourad PD. Increased anatomical specificity of neuromodulation via modulated focused ultrasound. PLoS One. 2014;9:e86939.

27. King RL, Brown JR, Newsome WT, Pauly KB. Effective parameters for ultrasound-induced in vivo neurostimulation. Ultrasound Med Biol. 2013;39:312-31.

28. Wahab RA, Choi M, Liu Y, Krauthamer V, Zderic V, Myers MR. Mechanical bioeffects of pulsed high intensity focused ultrasound on a simple neural model. Med Phys. 2012;39: 4274-83.

29. Krasovitski B, Frenkel V, Shoham S, Kimmel E. Intramembrane cavitation as a unifying mechanism for ultrasound-induced bioeffects. Proc Natl Acad Sci U S A. 2011;108:3258-63.

30. Plaksin M, Shoham S, Kimmel E. Intramembrane cavitation as a predictive bio-piezoelectric mechanism for ultrasonic brain stimulation. J Mol Neurosci. 2014;53:S103.

31. Tyler WJ. Noninvasive neuromodulation with ultrasound? A continuum mechanics hypothesis. Neuroscientist: A Rev J Bringing Neurobiol Neurol Psychiatry. 2011;17:25-36.

32. Kim H, Chiu A, Park S, Yoo SS. Image-guided navigation of single-element focused ultrasound transducer. Int J Imaging Syst Technol. 2012;22:177-84. 